Abstract 46 Table 1 Odds ratios and $95 \% \mathrm{Cl}$ from multivariate models of cutaneous lupus by smoking exposure in pack-years

\begin{tabular}{|c|c|c|c|c|c|c|}
\hline & Acute SLICCCutaneous & Chronic SLICC Cutaneous & Any SLICC Cutaneous & Any ACR Cutaneous & Any Muco-cutaneous* & Any SLICC-DI Skin Damage \\
\hline Nonsmoker (0 pk-yrs) & ref & ref & ref & ref & ref & ref \\
\hline Low (<5 pk-yrs) & $1.6(0.8-2.9)$ & $1.7(0.8-3.9)$ & $3.7(1.3-10.6)$ & $2.0(1.0-3.8)$ & $9.0(1.2-67.7)$ & $1.8(0.6-5.7)$ \\
\hline Med (5-10 pk-yrs) & $2.3(1.1-5.1)$ & $2.0(0.8-4.9)$ & $2.1(0.8-5.8)$ & $2.0(0.9-4.3)$ & $7.3(0.95-56.2)$ & $2.6(0.8-8.3)$ \\
\hline High (>10 pk-yrs) & $1.1(0.7-1.7)$ & $2.2(1.2-4.2)$ & $1.3(0.7-2.2)$ & $1.2(0.7-1.9)$ & $0.9(0.5-1.7)$ & $4.2(1.9-9.2)$ \\
\hline Early onset & $0.6(0.3-1.3)$ & $0.9(0.3-2.7)$ & $0.6(0.2-1.7)$ & $0.7(0.3-1.6)$ & $0.6(0.2-2.2)$ & $0.8(0.2-3.7)$ \\
\hline Usual onset & ref & ref & ref & ref & ref & ref \\
\hline Late onset & $0.3(0.1-0.8)$ & $1.0(0.3-3.0)$ & $0.3(0.1-0.97)$ & $0.5(0.2-1.1)$ & $0.4(0.1-1.6)$ & $1.3(0.3-6.4)$ \\
\hline Male & ref & ref & ref & ref & ref & ref \\
\hline Female & $2.6(1.5-4.7)$ & $1.1(0.5-2.6)$ & $4.6(2.6-8.1)$ & $2.5(1.4-4.4)$ & $5.2(2.9-9.4)$ & $1.1(0.4-3.4)$ \\
\hline White & ref & ref & ref & ref & ref & ref \\
\hline Black & $0.4(0.2-0.6)$ & $1.8(0.97-3.4)$ & $0.9(0.5-1.8)$ & $0.5(0.3-0.8)$ & $0.7(0.4-1.6)$ & $2.6(1.1-5.9)$ \\
\hline Other & $0.6(0.3-1.1)$ & $3.6(1.6-8.1)$ & $0.6(0.3-1.5)$ & $0.5(0.3-1.4)$ & $0.5(0.2-1.2)$ & $2.2(0.6-8.2)$ \\
\hline
\end{tabular}

Ref= referent category; *Any Mucocutaneous included any SLICC or ACR cutaneous or mucosal criteria.

compared to nonsmokers. Covariates included age category at diagnosis (early onset $<18$ years old, 18-50 years, or late onset $>50$ years), sex, and race. Analysis was performed using multivariate logistic regression to calculate odds ratios and 95\% confidence intervals (OR, (95\% CI)).

Results Among 631 SLE patients, mean age was 42, 91\% female, $82 \%$ white, and $40 \%$ ever smokers. Patients with low smoking exposure were nine times more likely to develop any mucocutaneous manifestations (OR 9.0, (1.2, 67.7)), four times more likely to meet any SLICC cutaneous criteria (OR 3.7, (OR 1.3, 10.6)), and twice as likely to meet ACR cutaneous criteria (OR $2.0(1.0,3.8)$ ) compared to non-smokers (table 1). Patients with medium smoking exposure were twice as likely to meet acute cutaneous SLICC criteria (OR 2.3, (1.1, 5.1)), whereas those with high smoking exposure had two-fold higher odds of discoid lupus (OR 2.1, (1.1, 4.1) data not shown). Chronic cutaneous SLICC criteria and DI cutaneous criteria showed linear pack-year trends that met significance with high smoking exposure (OR 2.2, (1.2, 4.2); OR 4.2, (0.9, 9.2)). Patients of color had increased risk for alopecia, discoid lupus, chronic cutaneous lupus, and DI skin damage. Limitations included sample size and just 18\% patients of color.

Conclusions Any smoking exposure was an independent risk factor for nearly all cutaneous SLE manifestations whereas high smoking exposure and patients of color had significantly increased risk of chronic cutaneous manifestations and skin damage. Findings suggest a dose relationship between smoking exposure and cutaneous manifestations/damage, making cessation an important strategy to potentially reduce disparities and improve cutaneous outcomes in SLE.

Funding Source(s): Support: Rheumatology Research Foundation

\section{TARGETED THERAPY USING INTRADERMAL INJECTION OF ETANERCEPT FOR REMISSION INDUCTION IN DISCOID LUPUS ERYTHEMATOSUS (TARGET-DLE): RESULTS FROM A PROOF-OF-CONCEPT PHASE II TRIAL}

\footnotetext{
${ }^{1}$ Md Yuzaiful Md Yusof, 'Miriam Wittmann, 'Catherine Fernandez, 'Duncan Wilson, ${ }^{2}$ Sara Edward, 'Giuseppina Abignano, ${ }^{1}$ Adewonuola Alase, ${ }^{2}$ Philip Laws, ${ }^{1}$ Mark Goodfield, ${ }^{1}$ Paul Emery, ${ }^{1}$ Edward Vital. ${ }^{1}$ University of Leeds; ${ }^{2}$ Leeds Teaching Hospitals NHS Trust
}

Background A significant proportion of patients with discoid lupus erythematosus (DLE) are resistant to conventional therapies. Tumour necrosis factor (TNF) is pathogenic in DLE. A concern with systemic TNF-i administration is induction of pathogenic autoantibodies and flare of disease. This could be overcome using a low-dose intra-dermal injection, which may be sufficient to neutralise the TNF in lesions, without systemic TNF effects.

The objective of this trial was to assess the efficacy and safety of a novel route of administration of a TNF-i using a low dose intra-dermal injection of etanercept (ETN) for remission induction in DLE.

Methods A prospective single arm, Simons 2-stage minimax design with Hybrid adaptation, phase II open label trial was conducted in Leeds [NCT02656082]. Key inclusion criteria were i) adults aged $1880 \mathrm{y}$; ii)oneactive DLE lesion and iii) refractory to anti-malarials. One index lesion with the highest activity was treated with weekly intra-dermal injection of up to $10 \mathrm{mg}$ ETN. The primary endpoint was 6 patients achieving the modified limited Score of Activity and Damage in DLE (ML-SADDLE) 20 response (defined as reduction $20 \%$ in total activity comprises erythema, induration and scaling from baseline) at Week 12 for a Phase 3 trial to be recommended. Secondary endpoints included change in objective outcome measures; lesional thermography and laser Doppler imaging.

Results All 25 DLE patients were recruited over 18 months (18 female, mean age $47 \pm 12$ y, 6 had SLE, 9 had positive ANA and median (range) no. of previous systemic therapies was 5 (116) 17 patients completed the primary
Abstract 47 Table 1 Secondary Endpoints (per protocol; $n=17$ )

\begin{tabular}{llll}
\hline Endpoint & $\begin{array}{l}\text { Pre- } \\
\text { Treatment }\end{array}$ & $\begin{array}{l}\text { Post- } \\
\text { Treatment }\end{array}$ & p-value \\
\hline Physician VAS, mean (SD) mm & $53.1(16)$ & $23.2(20)$ & $<0.001$ \\
Patient VAS, mean (SD) mm & $56.9(28)$ & $29.7(28)$ & 0.001 \\
DLQI, mean (SD) & $11.4(7)$ & $6.5(6)$ & $<0.001$ \\
Laser Doppler Imaging, mean (SD) perfusion unit & $495.1(224)$ & $376.2(223)$ & 0.018 \\
Infrared thermography, mean (SD), ${ }^{\circ}$ Celcius & $1.92(1.17)$ & $1.08(1.05)$ & 0.005 \\
\hline
\end{tabular}


efficacy assessment [Did not attend Week 12 visit $=1$, early withdrawals $=7$ (personal choice $=2, \mathrm{AE}=2$, worsening of $\mathrm{DLE}=1$, non-compliance $=1$, pregnant=1)]. The primary endpoint was met with 13/25 (52\%, 95\% CI 3173) meeting the ML-SADDLE 20 in full-set analysis. The rates for ML-SADDLE 50 and 70 were $48 \%$ and $20 \%$ respectively. Key secondary endpoints were met (table 1). Fifty-one AEs (treatment-emergent=28, Grade $3 / 4=4$ ) were recorded. There was no worsening of BILAG or SLEDAI in patients with SLE. Trough serum ETN levels were detected in 6/23 (26\%).

Conclusions Intradermal injection of ETN substantially reduced clinical activity, met its primary, secondary endpoints and was tolerable in DLE patients who were refractory to anti-malarials and other systemic therapies. This drug warrants further development in multi-centre trials. Analyses of other imaging and histological biomarkers are ongoing and can help stratifying patients for response.

Funding Source(s): National Institute of Health Research

\section{LOW-DOSE IL-2 THERAPY RESCUES DECREASED PERIPHERAL LYMPHOCYTES IN SLE PATIENTS WITH DIFFERENT INFECTIOUS STATUS}

'Sheng-xiao Zhang*, 'Jia Wang, 'Cai-hong Wang, 'Xiao-yan Wu, ${ }^{1}$ Jing Luo, 'Guangying Liu, ${ }^{1}$ Hong-yan Wen, ${ }^{2}$ Chong Gao, ${ }^{3}$ Xiao-feng Li. ${ }^{1}$ The Second Hospital of Shanxi Medical University; ${ }^{2}$ Department of Pathology,Brigham and Womens Hospital, Harvard Medical School; ${ }^{3}$ The Second Hospital of Shanxi Medical University

\subsection{6/lupus-2019-Ism.48}

Background Systemic lupus erythematosus (SLE) is a heterogeneous autoimmune disorder characterized by autoantibody production to a variety of self-antigens. This abnormal immunological background and immune-suppressive therapies predispose SLE patients to infection. Recent studies have revealed that low-dose IL-2 not only regulates immune balance but also alleviates directly SLE disease activity. In this study, we assessed the alterations of lymphocyte subpopulations and the effect of restore the immunologic balances by low dose IL-2 in SLE patients with different infectious status.

\section{(A)}

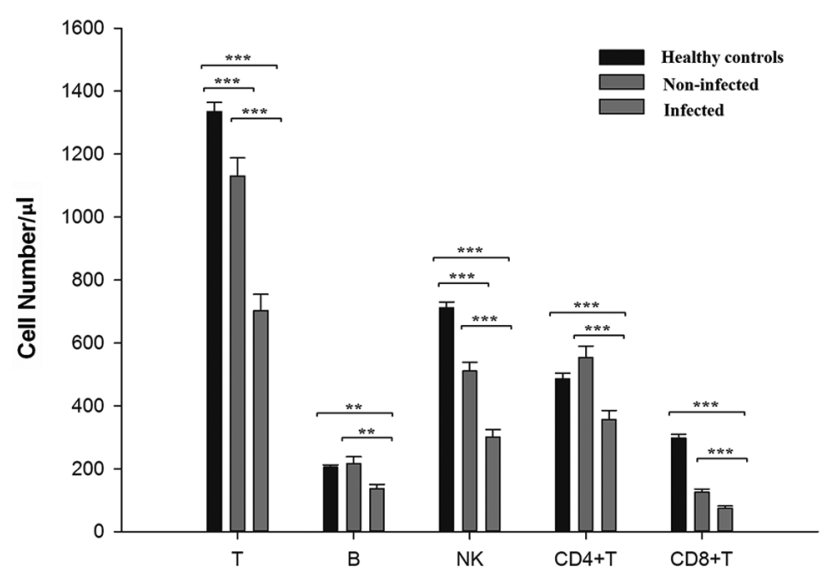

(C)

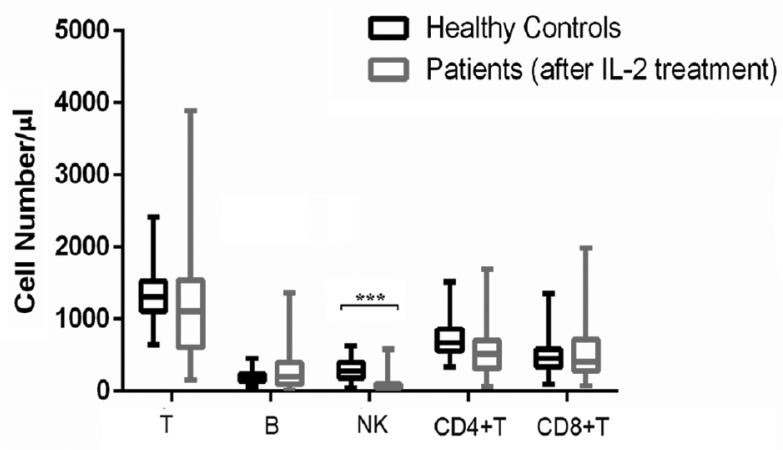

(B)

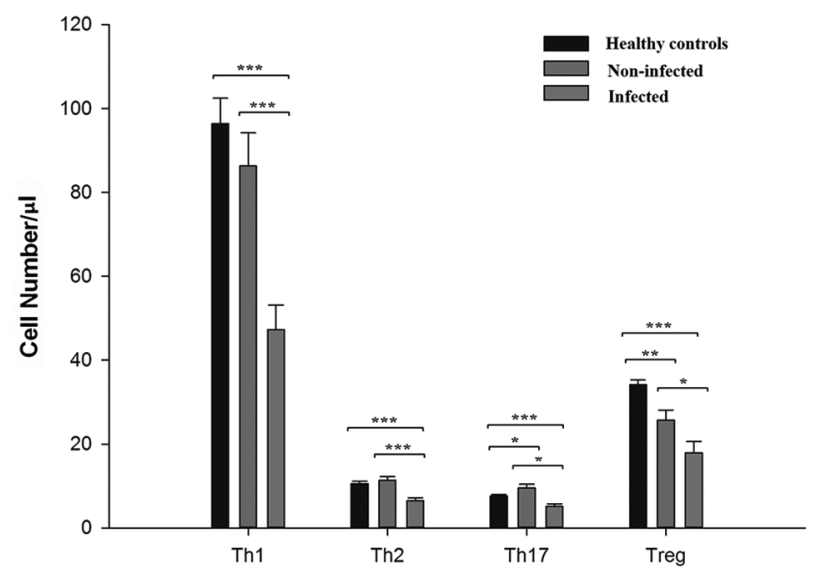

(D)

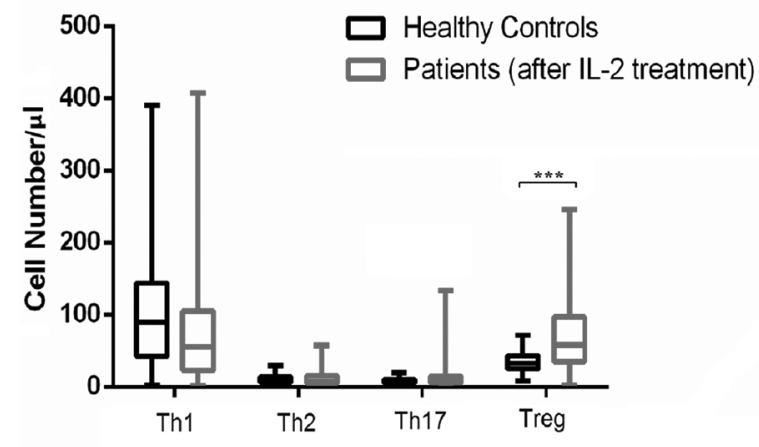

Abstract 48 Figure 1 Multiple subpopulations of lymphocytes decreased in peripheral blood of SLE patients with infection and IL-2 up-regulated lymphocytes in patients with SLE near to the levels of healthy controls. Absolute numbers of peripheral lymphocytes subpopulations were analyzed by flow cytometry. (A) SLE patients $(n=495)$ had lower levels of T, B, NK, CD4+T and CD8+T cells in PB compared with that of health controls. These cells in the infected groups $(n=162)$ were also much lower than those in non-infected patients $(n=333)$. (B) In CD4+T cells, patients with SLE had lower levels of Th1 cells, Th2 cells, Th17 cells as well as Treg cells compared with those in health donors ( $n=132)$, especially the infected groups. (C) IL-2 expanded T, B, CD4+T and CD8+T cells in PB of SLE patients $(n=54)$ even near to the levels of healthy controls $(n=132)$. (D) In CD4+T cell subsets, IL-2 restored Th1, Th2, Th17 and Tregs of SLE patients to the comparable levels of those of health donors. Importantly, Tregs were significantly increased, especially the infected groups. ${ }^{*} \mathrm{P}<0.05,{ }^{*} \mathrm{P}<0.01,{ }^{*}{ }^{*} \mathrm{P}<0.001$. 